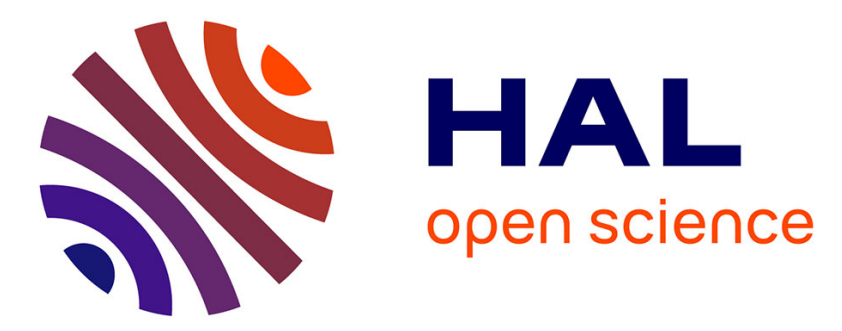

\title{
Modelling of structural domains and elastic strain calculation in rhombohedral La1-xSrxMnO3 films on (110) $\mathrm{SrTiO3}$
}

Nayel Farag, Manfred Bobeth, Wolfgang Pompe, Alexei E. Romanov

\section{- To cite this version:}

Nayel Farag, Manfred Bobeth, Wolfgang Pompe, Alexei E. Romanov. Modelling of structural domains and elastic strain calculation in rhombohedral La1-xSrxMnO3 films on (110) SrTiO3. Philosophical Magazine, 2006, 87 (06), pp.823-842. 10.1080/14786430600993331 . hal-00513774

\section{HAL Id: hal-00513774 \\ https://hal.science/hal-00513774}

Submitted on 1 Sep 2010

HAL is a multi-disciplinary open access archive for the deposit and dissemination of scientific research documents, whether they are published or not. The documents may come from teaching and research institutions in France or abroad, or from public or private research centers.
L'archive ouverte pluridisciplinaire HAL, est destinée au dépôt et à la diffusion de documents scientifiques de niveau recherche, publiés ou non, émanant des établissements d'enseignement et de recherche français ou étrangers, des laboratoires publics ou privés. 


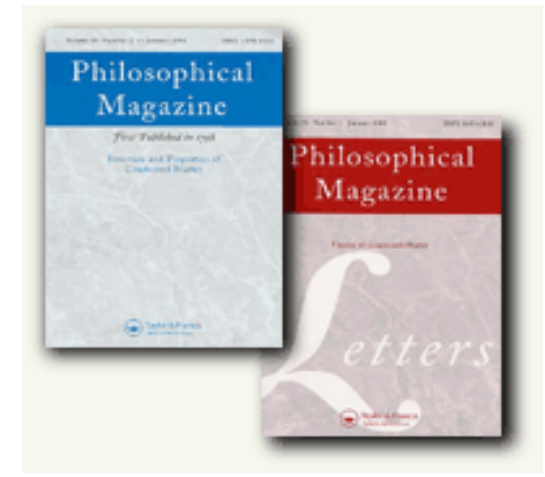

\section{Modelling of structural domains and elastic strain calculation in rhombohedral $\mathrm{La}_{1-x} \mathrm{Sr}_{x} \mathrm{MnO}_{3}$ films on (110) $\mathrm{SrTiO}_{3}$}

\begin{tabular}{|r|l|}
\hline Journal: & Philosophical Magazine \& Philosophical Magazine Letters \\
\hline Manuscript ID: & TPHM-06-May-0175.R1 \\
\hline Journal Selection: & Philosophical Magazine \\
\hline Date Submitted by the \\
Author: & 01-Sep-2006 \\
\hline Keywlete List of Authors: & $\begin{array}{l}\text { Farag, Nayel; Technische Universität Dresden, Institut für } \\
\text { Werkstoffwissenschaft } \\
\text { Bobeth, Manfred; Technische Universität Dresden, Institut für } \\
\text { Werkstoffwissenschaft } \\
\text { Pompe, Wolfgang; Technische Universität Dresden, Institut für } \\
\text { Werkstoffwissenschaft } \\
\text { Romanov, Alexei; Russian Academy of Sciences, Ioffe Physico- } \\
\text { Technical Institute }\end{array}$ \\
\hline Keywords (user supplied): & $\begin{array}{l}\text { lislocation mechanics, domain structure, epitaxial systems, } \\
\text { manganites, micromechanics, strain, structural transitions, twinning } \\
\text { manganites }\end{array}$ \\
\hline
\end{tabular}

\section{今 scholaroNE" \\ Manuscript Central}


Page 1 of 8Philosophical Magazine \& Philosophical Magazine Letters
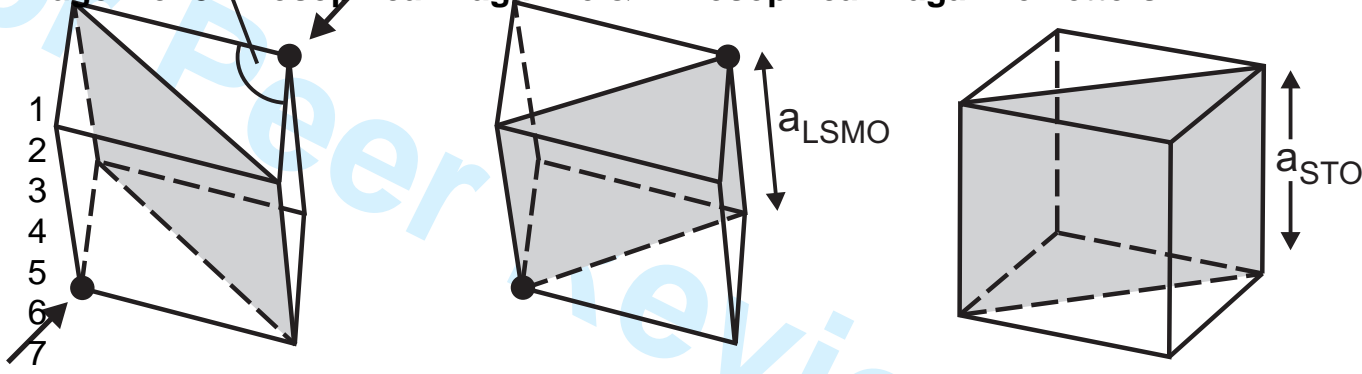

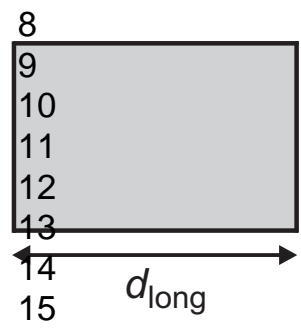

16

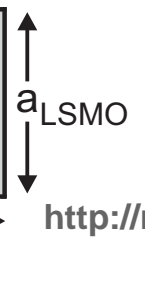

(a)

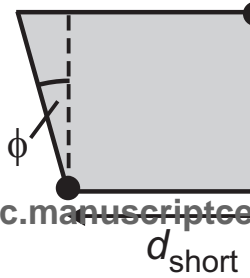

(b)

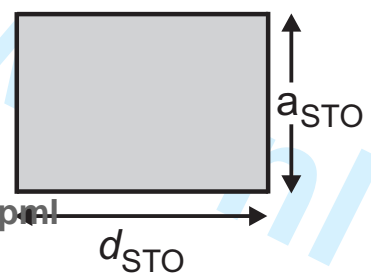

(c) 


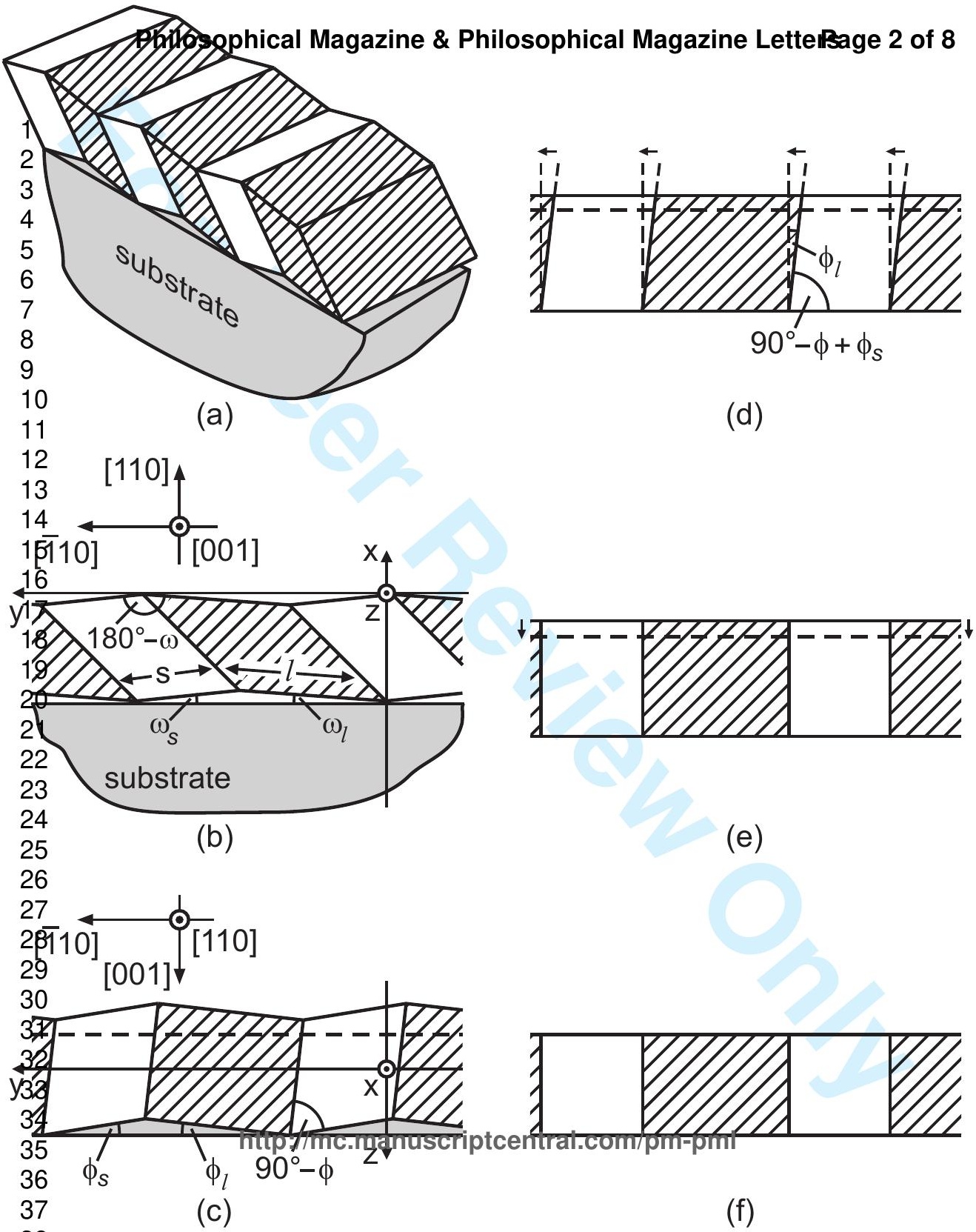




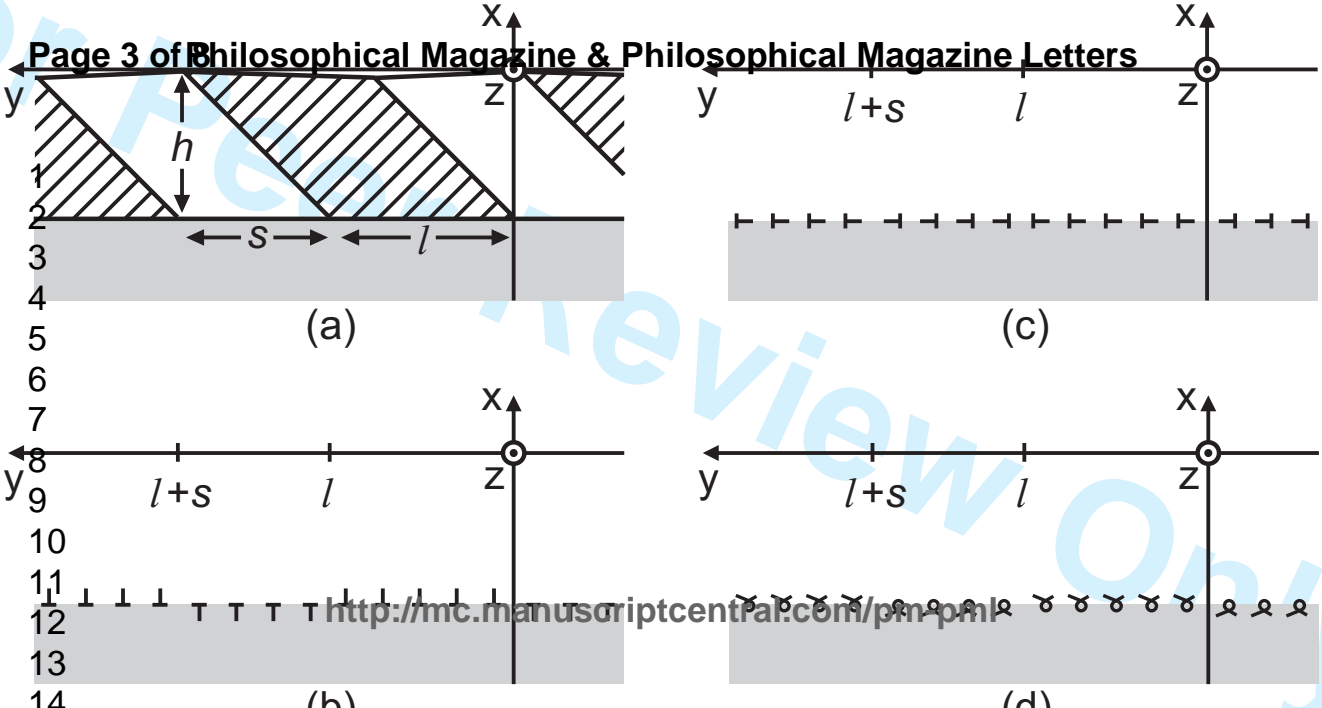

(a)

14 



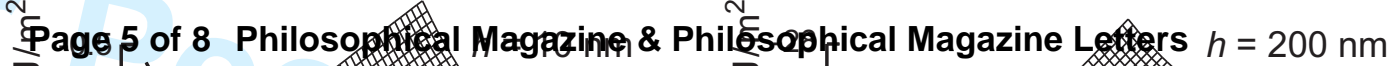

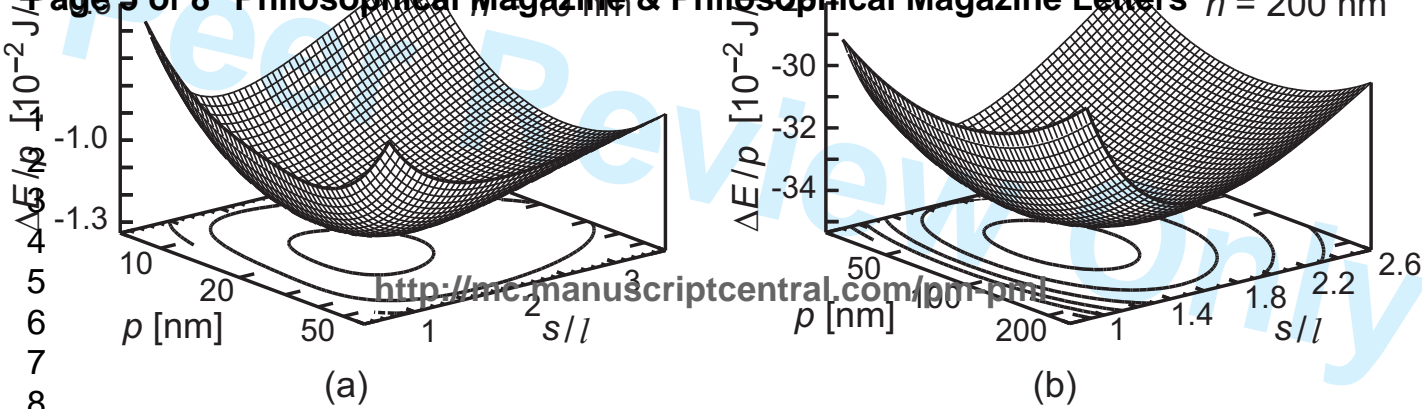

8

(a)

(b) 


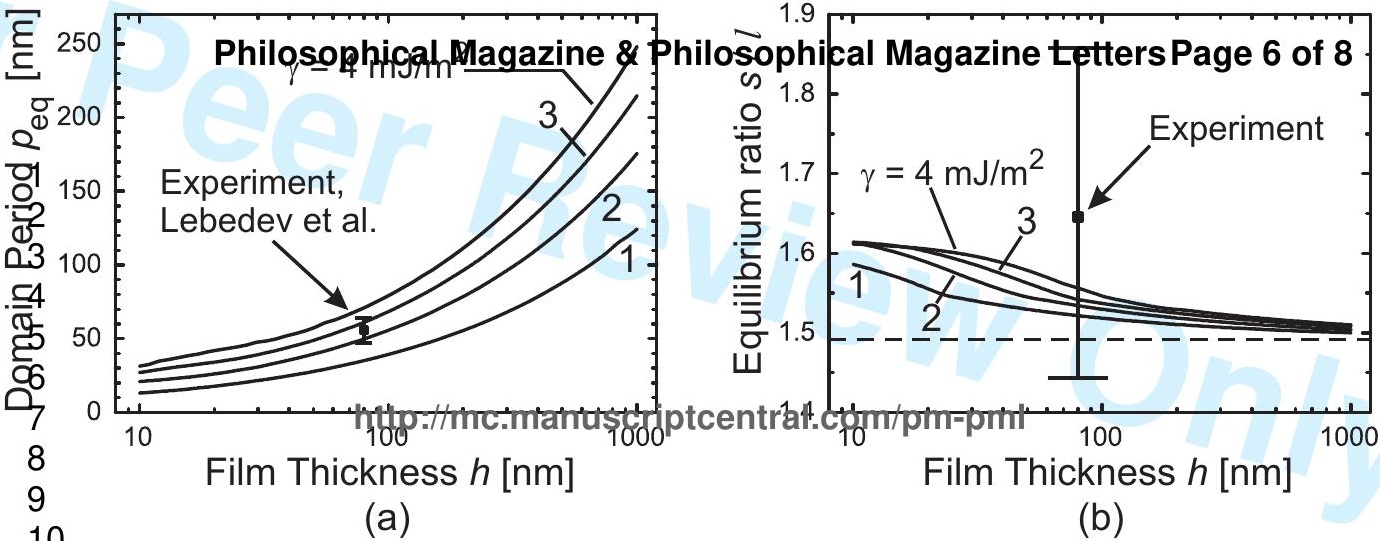




\subsection{1 י י}

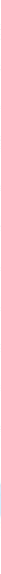




\section{Philosophical Magazine \& Philosophical Magazine Rotgers of 8}

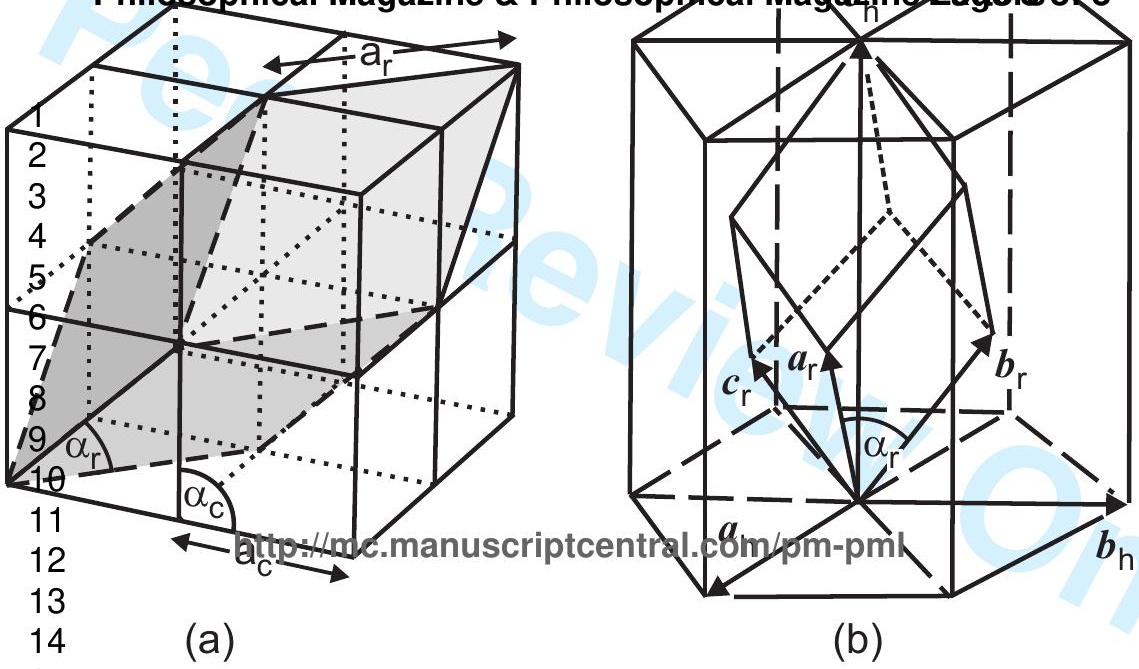

